\title{
APPENDIX TO \\ THE CONTRIBUTION OF STRUCTURAL BREAK MODELS TO FORECASTING MACROECONOMIC SERIES
}

\author{
Luc Bauwens $^{1}$, Gary Koop ${ }^{2}$, Dimitris Korobilis ${ }^{1}$, and Jeroen V.K. Rombouts ${ }^{3,1}$
}

July 5,2011

This document contains four appendices to the paper of Bauwens, Koop, Korobilis, and Rombouts (2011). The first three appendices contain details about the implementation of the estimation and forecasting of the structural break models named PPT and KP in the paper. These models are explained in Section 2 of the paper and information about the forecasting implementation of these models is presented in Section 4 of the paper. The fourth appendix contains tables that show detailed results that are summarized and discussed in Section 5 of the paper.

\footnotetext{
${ }^{1}$ Université catholique de Louvain, CORE, B-1348 Louvain-La-Neuve.

${ }^{2}$ University of Strathclyde.

${ }^{3}$ Institute of Applied Economics at HEC Montréal, CIRANO, CIRPEE).

This research was supported by the ESRC under grant RES-062-23-2646, and by the contract "Projet d'Actions de Recherche Concertées" 07/12-002 of the "Communauté francaise de Belgique", granted by the "Académie universitaire Louvain". This text presents research results of the Belgian Program on Interuniversity Poles of Attraction initiated by the Belgian State, Prime Minister's Office, Science Policy Programming.
} 


\section{Appendix A: Technical Details for PPT Approach}

In this appendix, we describe posterior and predictive simulation as well as prior elicitation for our implementation of the PPT approach. Bauwens and Rombouts (2010) provide more details for posterior simulation and computing the marginal likelihood, which is used for choosing the number of breaks.

The model is defined by $y_{t}=Z_{t} \beta_{s_{t}}+\sigma_{s_{t}} \varepsilon_{t}$ and by the break process which involves $S^{T}=\left(s_{1}, . ., s_{T}\right)^{\prime}$ where $s_{t} \in\{1,2, . ., K\}$ is a state variable and $K$ is the number of in sample regimes (see Section 2 of the paper). Notice that the last regime is an absorbing state over the sample period, but PPT relax this in the forecast period.

\section{Priors}

We use priors of the form:

$$
\begin{aligned}
\beta_{j} \mid \beta_{0}, B_{0} & \sim N_{m}\left(\beta_{0}, B_{0}\right), \\
\beta_{0} & \sim N_{m}\left(\underline{\mu}_{\beta}, \underline{V}_{\beta}\right), \\
B_{0}^{-1} & \sim \operatorname{Wishart}(\underline{\xi}, \underline{B}), \\
\sigma_{j}^{-2} \mid v_{0}, d_{0} & \sim \operatorname{Gamma}\left(v_{0}, d_{0}\right), \\
v_{0} & \sim \operatorname{Gamma}(\underline{\lambda}, \underline{\rho}), \\
d_{0} & \sim \operatorname{Gamma}(\underline{c}, \underline{d}) \\
p_{i} & \sim \operatorname{Beta}(\underline{a}, \underline{b}) .
\end{aligned}
$$

In particular, in the forecasting exercise we set $\underline{\mu}_{\beta}=0, \underline{V}_{\beta}=I_{m}, \underline{B}=10 I_{m}, \underline{\xi}=m+1$ (where $m$ is the dimension of $Z_{t}$ ), $\underline{\lambda}=1, \underline{\rho}=0.1, \underline{c}=1, \underline{d}=0.1$, and $\underline{a}=\underline{b}=1$. This implies that all priors are proper but little informative.

\section{Posterior simulator}

The posterior simulation algorithm is a Gibbs sampler. Given initial conditions, the data, and in each block the other parameters, the sampling is done as follows:

1. Draw $S^{T}$ using Chib's (1998) algorithm.

2. Draw $p_{i}$ from Beta $\left(\underline{a}+T_{i}, \underline{b}+1\right)$ for $i=1, \ldots, K$, where $T_{i}$ is the number of observations in regime $i$.

3. Draw $\beta_{i} \mid \sigma_{i}^{2}$ from Normal and $\sigma_{i}^{2} \mid \beta_{i}$ from Gamma, for $i=1,2, \ldots, K$.

4. Draw $\beta_{0} \mid B_{0}$ from Normal and $B_{0}^{-1} \mid \beta_{0}$ from Wishart.

5. Draw $d_{0} \mid v_{0}$ from Gamma and $v_{0} \mid d_{0}$ by numerical evaluation and inversion of its cdf. 


\section{Appendix B: Technical Details for KP Approach}

In this appendix, we describe posterior and predictive simulation as well as prior elicitation for our implementation of the KP approach.

It is convenient to write the model equation as $y_{t}=Z_{t} \beta_{s_{t}}+\exp \left(\omega_{s_{t}} / 2\right) \varepsilon_{t}$. The transition probabilities between the states are defined in equation (5) of the paper so that the last diagonal element of the transition matrix is equal to $p_{K}$ rather than one as in the PPT approach.

\section{Priors}

We use priors of the form:

$$
\begin{aligned}
\beta_{j} & \sim N_{m}\left(\beta_{j-1}, B_{0}\right) \\
\omega_{j} & \sim N\left(\omega_{j-1}, \delta\right) \\
\beta_{0} & \sim N_{m}\left(0, \underline{V}_{\beta}\right) \\
\omega_{0} & \sim N\left(0, \underline{V}_{\omega}\right) \\
B_{0}^{-1} & \sim \text { Wishart }(\underline{\xi}, \underline{B}) \\
\delta^{-1} & \sim \operatorname{Gamma}\left(\underline{\kappa_{1}}, \underline{\kappa_{2}}\right) \\
p_{i, i} & \sim \operatorname{Beta}(\underline{a}, \underline{b}) .
\end{aligned}
$$

In particular, in the forecasting exercise we set $\underline{V}_{\beta}=I_{m}, \underline{V}_{\omega}=1, \underline{B}=10 I_{m}, \underline{\xi}=m+1$, $\underline{\kappa_{1}}=\underline{\kappa_{2}}=0.5$, and $\underline{a}=\underline{b}=1$. This implies that all priors are proper but very little informative.

\section{Posterior simulator}

The posterior simulation algorithm is a Gibbs sampler. Given initial conditions, the data, and in each block the other parameters, the sampling is done as follows:

1. Draw $S^{T}$ using Chib's (1998) algorithm.

2. Draw $p_{i}$ from Beta $\left(\underline{a}+T_{i}, \underline{b}+1\right)$ for $i=1, \ldots, K$, where $T_{i}$ is the number of observations in regime $i$.

3. Draw $\left[\beta_{s_{t}}\right]_{t=1}^{T}$ using the modified Kalman filter algorithm (see below).

4. Draw $\left[\omega_{s_{t}}\right]_{t=1}^{T}$ using the modified Kalman filter algorithm, after writing the model in appropriate linear state space form using the Kim, Shephard and Chib (1998) algorithm.

5. Draw $B_{0}^{-1}$ and $\delta^{-1}$, conditional on the draws of $\beta_{t}$ and $\omega_{t}$, using standard expressions.

\section{Modified Kalman filter algorithm}

Consider a state-space model of the following form:

$$
\begin{aligned}
& y_{t}=z_{t} a_{s t}+\varepsilon_{t} \\
& a_{j}=a_{j-1}+\eta_{s_{t}} \\
& \varepsilon_{t} \sim N\left(0, \gamma_{1}^{2}\right), \eta_{j} \sim N\left(0, \gamma_{2}^{2}\right)
\end{aligned}
$$


conditional on knowing $s_{t}$, where (1a) is the measurement equation and (1b) is the state equation, with observed data $y_{t}$ and unobserved state $a_{s_{t}}$. If the errors $\epsilon_{t}, \eta_{t}$ are iid and uncorrelated with each other, we can use the Kalman filter to estimate the state $a$.

Let $a_{t \mid s}$ denote the expected value of $a_{t}$ and $P_{t \mid s}$ its corresponding variance, using data up to time $s$. Given starting values $a_{0 \mid 0}$ and $P_{0 \mid 0}$, the Kalman filter recursions provide us with initial filtered estimates:

$$
\begin{aligned}
a_{t \mid t-1} & =a_{t-1 \mid t-1} \\
P_{t \mid t-1} & =\left\{\begin{array}{c}
P_{t-1 \mid t-1}+\gamma_{2}^{2}, \text { if } s_{t-1} \neq s_{t} \\
P_{t-1 \mid t-1}, \text { otherwise }
\end{array}\right. \\
K_{t} & =P_{t \mid t-1} z_{t}^{\prime}\left(z_{t} P_{t \mid t-1} z_{t}+\gamma_{1}^{2}\right)^{-1} \\
a_{t \mid t} & =a_{t \mid t-1}+K_{t}\left(y_{t}-z_{t} a_{t \mid t-1}\right) \\
P_{t \mid t} & =P_{t \mid t-1}-K_{t} z_{t} P_{t \mid t-1} .
\end{aligned}
$$

Once we reach the last period $(t=T)$ we take the standard draw $a_{s_{T}} \sim N\left(a_{T \mid T}, P_{T \mid T}\right)$. If $s_{T}=T$ then a break occurs in each observation and we have a full tvp model, so that the Carter and Kohn smoother applies to all observations $t$. However with structural breaks models it will be the case that $s_{T}<<T$ (i.e. the number of breaks is smaller than the number of observations, i.e. we do not have a full tvp model), we can only simulate $a_{j}$ for $j=s_{T}+1, \ldots, T$ (i.e. the "out-of-sample breaks" in $a$ ) using equation (1b). For $j=1, \ldots, s_{T}$ we can use a standard smoother to get smoothed estimates. To do that, we run the backward recursions for $t=T-1, \ldots, 1$ :

$$
\begin{aligned}
& a_{t \mid t+1}=a_{t \mid t}+P_{t \mid t} P_{t+1 \mid t}^{\prime}\left(a_{t+1}-a_{t \mid t}\right), \text { iff } s_{t+1} \neq s_{t} \\
& P_{t \mid t+1}=P_{t \mid t}-P_{t \mid t} P_{t+1 \mid t}^{\prime} P_{t \mid t}, \text { iff } s_{t+1} \neq s_{t}
\end{aligned}
$$

and draw $a_{s_{t}} \sim N\left(a_{t \mid t+1}, P_{t \mid t+1}\right)$ iff $s_{t+1} \neq s_{t}$. 


\section{Appendix C: Predictive Simulator for PPT and KP models}

\section{Forecasting with no breaks out-of-sample (PPT model)}

Since the PPT model implies that observations following $T$ (the last sample date) are generated from $y_{T+h} \mid Y_{T+h-1}, \theta_{K}$ where $\theta_{K}=\left(\beta_{K}, \sigma_{K}^{2}\right)$, i.e. under the last operating regime, we can compute predictive densities as follows:

$$
\begin{aligned}
& p\left(y_{T+h} \mid s_{T+h}=K, s_{T}=K, Y_{T}\right)=\int \ldots \int \prod_{j=0}^{h-1} p\left(y_{T+h-j} \mid Y_{T+h-1-j}, \theta_{K}\right) \\
& p\left(\theta_{K} \mid \theta_{0}, S_{T}, Y_{T}\right) p\left(\theta_{0} \mid S_{T}, Y_{T}, \underline{A}\right) p\left(S_{T} \mid Y_{T}\right) d y_{T+h-1} \ldots d y_{T+1} d \theta_{K} d \theta_{0} d S_{T-1},
\end{aligned}
$$

where the integration is done with respect to $S_{T-1}$ rather than $S_{T}$ since $s_{T}=K$. This is implemented by simulation within the Gibbs sampler for the posterior density: for each Gibbs draw of $\theta_{K}, \theta_{0}$ and $S_{T-1}$, we generate sequentially future values $y_{T+1}, \ldots, y_{T+h}$, each from $y_{T+h-j} \sim p\left(y_{T+h-j} \mid Y_{T+h-1-j}, \theta_{K}\right)$, and we keep $y_{T+h}$ as a draw of the corresponding predictive density $p\left(y_{T+h} \mid s_{T+h}=K, s_{T}=K, Y_{T}\right)$. Doing this for e.g. $h=4$ provides also the draws of the predictive densities for $h \leq 4$.

\section{Forecasting with breaks out-of-sample (PPT \& KP models)}

The previous discussion does not allow for a break to occur in the forecast period. In order to allow in the PPT for the possibility of occurrence of one new regime after $T$, we lift the restriction $p_{K}=1$ (something already done in the $\mathrm{KP}$ model) and extend the transition matrix to

$$
\left(\begin{array}{ccccccc}
p_{1} & 1-p_{1} & 0 & \ldots & 0 & 0 & 0 \\
0 & p_{2} & 1-p_{2} & \ldots & 0 & 0 & 0 \\
\vdots & \vdots & \vdots & \vdots & \vdots & \vdots & \vdots \\
0 & 0 & 0 & \ldots & p_{K-1} & 1-p_{K-1} & 0 \\
0 & 0 & 0 & \ldots & 0 & p_{K} & 1-p_{K} \\
0 & 0 & 0 & \ldots & 0 & 0 & 1
\end{array}\right) .
$$

Additional regimes can be added by extending further the transition matrix, but here we consider the predictive density subject to one break occurring after date $T$. Assume that the break occurs at date $T+d$ where $d$ can take any value in the set $\{1,2, \ldots, h\}$. For the predictive simulation of $y_{T+h}$ with $h<d$ (the no-post-sample break case), we proceed as above. For $h \geq d$, the break occurrence implies that $y_{T+h} \sim p\left(y_{T+h} \mid Y_{T+h-1}, \theta_{K+1}\right)$ where $\theta_{K+1}$ is the parameter characterizing the new regime, and is drawn from its hierarchical prior density $p\left(\theta_{K+1} \mid \theta_{0}\right)$. The observed sample does not provide information about $\theta_{K+1}$ and thus does not directly update this prior, but it does so indirectly by updating the prior information about $\theta_{0}$ since this is drawn from its posterior distribution in the Gibbs sampler.

Assume first that $h=d=1$. Then, given $\theta_{0}$ (drawn in the Gibbs sampler), $\theta_{K+1}$ is drawn from $p\left(\theta_{K+1} \mid \theta_{0}\right)$ and given this draw, $y_{T+1}$ is drawn from $p\left(y_{T+1} \mid Y_{T}, \theta_{K+1}\right)$. This procedure is repeated at each iteration of the Gibbs sampler and delivers a sample of draws from the predictive density $p\left(y_{T+1} \mid s_{T+1}=K+1, s_{T}=K, Y_{T}\right)$.

Next assume that $h=2$ and $d=1: y_{T+1}$ is simulated as explained just above, and $y_{T+2}$ is drawn from $p\left(y_{T+2} \mid y_{T+1}, Y_{T}, \theta_{K+1}\right)$ where $y_{T+1}$ is set at its simulated value and $\theta_{K+1}$ is maintained to be the value used for drawing this $y_{T+1}$. For $h$ larger than 2, one proceeds sequentially in the same way, i.e. freezing $\theta_{K+1}$ and using the simulated lagged values $y_{T+h-j}$ $(j=1,2, \ldots, h-1)$ in the conditioning of $p\left(y_{T+h} \mid Y_{T+h-1}, \theta_{K+1}\right)$. 
Finally if $h \geq d \geq 1$, the values $y_{T+j}$ for $j=1,2, \ldots, d-1$ are sequentially simulated as in the no-post-sample break case. Then for $j=d, \theta_{K+1}$ is drawn from $p\left(\theta_{K+1} \mid \theta_{0}\right)$ and given this draw, $y_{T+j}$ for $j=d, d+1, \ldots, h$ are drawn sequentially. The next formula validates this simulation procedure for known break date $\mid \tau_{K}$ equal to $T+d$ :

$$
\begin{aligned}
& p\left(y_{T+h} \mid \tau_{K}=T+d, s_{T+h}=K+1, s_{T}=K, Y_{T}\right)= \\
& \int \ldots \int \prod_{j=0}^{h-1} p\left(y_{T+h-j} \mid Y_{T+h-1-j}, \theta_{K+1} 1_{\{h \geq d\}}+\theta_{K} 1_{\{h<d\}}\right) \\
& p\left(\theta_{K+1} \mid \theta_{0}, S_{T}, Y_{T}\right) p\left(\theta_{K} \mid \theta_{0}, S_{T}, Y_{T}\right) p\left(\theta_{0} \mid S_{T}, Y_{T}, \underline{A}\right) p\left(S_{T} \mid Y_{T}\right) \\
& d y_{T+h-1} \ldots d y_{T+1} d \theta_{K+1} d \theta_{K} d \theta_{0} d S_{T-1}
\end{aligned}
$$

where $1_{\{h \geq d\}}$ is equal to 1 if $h \geq d$ and 0 otherwise, and $1_{\{h<d\}}=1-1_{\{h \geq d\}}$. To marginalize this density with respect to the break date $d$, we sum over all values of $d$ as follows: $p\left(y_{T+h} \mid s_{T+h}=K+1, s_{T}=K, Y_{T}\right)=$

$$
\begin{array}{rl}
\sum_{d=1}^{h} & p\left(y_{T+h} \mid \tau_{K}=T+d, s_{T+h}=K+1, s_{T}=K, Y_{T}\right) \\
& \times \operatorname{Pr}\left[\tau_{K}=T+d \mid s_{T+h}=K+1, s_{T}=K, Y_{T}\right]
\end{array}
$$

with $\operatorname{Pr}\left[\tau_{K}=T+d \mid s_{T+h}=K+1, s_{T}=K, Y_{T}\right]=p_{K}^{d-1}\left(1-p_{K}\right) /\left(1-p_{K}^{h}\right)$. Finally, we can integrate $p\left(y_{T+h} \mid s_{T+h}, s_{T}=K, Y_{T}\right)$ with respect to the number of post-sample breaks (0 or 1): $p\left(y_{T+h} \mid s_{T}=K, Y_{T}\right)=$

$$
\begin{aligned}
& p\left(y_{T+h} \mid s_{T+h}=K, s_{T}=K, Y_{T}\right) p\left(s_{T+h}=K \mid s_{T}=K, Y_{T}\right) \\
& p\left(y_{T+h} \mid s_{T+h}=K+1, s_{T}=K, Y_{T}\right)\left[1-p\left(s_{T+h}=K \mid s_{T}=K, Y_{T}\right)\right]
\end{aligned}
$$

where $p\left(s_{T+h}=K \mid s_{T}=K, Y_{T}\right)=p_{K K}^{h}$. This is simulated by drawing $s_{T+h}$ from its discrete distribution, and then $y_{T+h}$ from (4) if $s_{T+h}=K$ and from (6) if $s_{T+h}=K+1$. To sample the discrete distribution, we need a value of $p_{K}$. This is simulated in the Gibbs sampler from its full conditional posterior density, which is $\operatorname{Beta}\left(\underline{a}+T_{K}, \underline{b}+1\right)$, where $T_{K}$ is the number of observations in regime $K$ according to the sampled $S_{T}$ vector.

As an example, to implement the simulation of $y_{T+1}$, we substitute (4), (6) and (5) in (7) and obtain $p\left(y_{T+1} \mid s_{T}=K, Y_{T}\right)=$

$$
\begin{array}{r}
p_{K} \int \ldots \int p\left(y_{T+1} \mid Y_{T}, \theta_{K}\right) p\left(\theta_{K} \mid \theta_{0}, S_{T}, Y_{T}\right) p\left(\theta_{0} \mid S_{T}, Y_{T}, \underline{A}\right) p\left(S_{T} \mid Y_{T}\right) \\
d \theta_{K} d \theta_{0} d S_{T-1} \\
+\left(1-p_{K}\right) \int \ldots \int p\left(y_{T+1} \mid Y_{T}, \theta_{K+1}\right) p\left(\theta_{K+1} \mid \theta_{0}, S_{T}, Y_{T}\right) p\left(\theta_{K} \mid \theta_{0}, S_{T}, Y_{T}\right) \\
p\left(\theta_{0} \mid S_{T}, Y_{T}, \underline{A}\right) p\left(S_{T} \mid Y_{T}\right) d \theta_{K+1} d \theta_{K} d \theta_{0} d S_{T-1} .
\end{array}
$$

This formula shows that the simulation for one predictive draw in the KP model, and the PPT model with the possibility of breaks occurring out-of-sample, is performed as follows:

1. Draw $S_{T}, \theta_{0}$ and $\theta_{K}$ from the posterior (i.e. use a draw of the Gibbs sampler once it has converged).

2. Draw $p_{K} \sim \operatorname{Beta}\left(\underline{a}+T_{K}, \underline{b}+1\right)$.

3. Draw $s_{T+1}=K$ or $K+1$ with respective probabilities $\left(p_{K}, 1-p_{K}\right)$.

4. If $s_{T+1}=K$, draw $y_{T+1} \sim p\left(y_{T+1} \mid Y_{T}, \theta_{K}\right)$. If $s_{T+1}=K+1$, draw $\theta_{K+1} \sim p\left(\theta_{K+1} \mid \theta_{0}, S_{T}, Y_{T}\right)$ and $y_{T+1} \sim p\left(y_{T+1} \mid Y_{T}, \theta_{K+1}\right)$.

If this is repeated as many times as one iterates in the Gibbs sampler, one obtains as many draws of the predictive of $y_{T+1}$. Generalizing this algorithm to $h \geq 2$ is not difficult but requires lengthy formulas. 


\section{Appendix D: Additional Tables}

These tables are providing detailed results on which Tables 5 and 6 in the paper are based. For each series in Table 1 of the paper, we provide in the following tables the RMSE and APL values from the recursive forecasting exercise described in Section 5 of the paper. For one-step ahead forecasts, see Tables 1 (RMSE) and 3 (APL) and for four-step ahead forecasts see Tables 2 and 4. We report the relative values, with the model in the last column (UC-SV) serving as reference. This reference model is just chosen for convenience.

The RMSE/APL values for the reference model are reported to fix their order of magnitude. For example, in Table 1, we see that for the UC-SV model and the first series, the RMSE is equal to 0.608, whereas the relative RMSE for PPT10 is 0.989 , implying that PPT11 has a RMSE 1.1 percent lower than the UC-SV model. For each series, the smallest (for RMSE) or largest (for APL) value across all models is in bold. If this global minimum is in the set of break models, the value in italics is the minimum across the no-break models (see Table 2 of the paper for the definition of break and no-break models). If the global minimum is in the latter group, the value in italics is the minimizer across the break models. 


\begin{tabular}{|c|c|c|c|c|c|c|c|c|c|c|c|c|c|c|c|}
\hline & $\begin{array}{l}\text { series } \\
\text { DPCog }\end{array}$ & $\begin{array}{l}\text { PPT10 } \\
P P^{2}\end{array}$ & PPT11 & $\begin{array}{l}\text { PPT40 } \\
P 047\end{array}$ & PPT41 & KP1 & KP4 & TVP1 & TVP4 & ROW1 & ROW4 & REC1 & REC4 & UC-SV & \\
\hline$\overline{1}$ & GDPC96 & 0.989 & 0.991 & 0.947 & 0.945 & 0.994 & 0.967 & 0.999 & 0.965 & 1.048 & 1.019 & 1.007 & 0.964 & 0.608 & 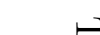 \\
\hline 2 & GDPDEF & 1.015 & 1.011 & 0.969 & 0.964 & 1.075 & 0.983 & 1.000 & 0.944 & 1.021 & 1.058 & 1.082 & 0.969 & 0.261 & \\
\hline 3 & PCECC96 & 1.139 & 1.135 & 0.962 & 0.965 & 1.126 & 0.983 & 1.157 & 1.024 & 1.070 & 0.997 & 1.137 & 0.989 & 0.419 & \\
\hline 4 & PCECTPI & 1.027 & 1.030 & 1.007 & 1.003 & 1.029 & 1.029 & 0.942 & 0.963 & 0.953 & 0.969 & 1.037 & 1.008 & 0.439 & \\
\hline 5 & GPDIC96 & 1.011 & 1.017 & 0.996 & 0.998 & 1.006 & 0.999 & 1.024 & 1.009 & 1.011 & 1.043 & 1.018 & 0.999 & 0.354 & \\
\hline $6^{*}$ & OPHPBS & 0.831 & 0.836 & 0.804 & 0.802 & 0.844 & 0.803 & 0.858 & 0.837 & 0.900 & 0.911 & 0.831 & 0.800 & 0.844 & 8 \\
\hline 7 & ULCNFB & 0.896 & 0.898 & 0.824 & 0.823 & 0.941 & 0.829 & 0.800 & 0.804 & 0.813 & 0.826 & 0.930 & 0.827 & 1.275 & \\
\hline 8 & CPIAUCSL & 0.702 & 0.700 & 0.725 & 0.719 & 0.689 & 1.253 & 0.699 & 0.675 & 0.714 & 0.707 & 0.698 & 0.676 & 0.986 & \\
\hline 9 & PPIFCG & 0.730 & 0.730 & 0.705 & 0.698 & 0.721 & 1.301 & 0.722 & 0.671 & 0.754 & 0.728 & 0.729 & 0.677 & 2.499 & \\
\hline 10 & TB3MS & 0.937 & 0.939 & 0.936 & 0.936 & 0.919 & 1.004 & 1.033 & 1.019 & 0.976 & 1.057 & 1.051 & 1.068 & 0.381 & \\
\hline 11 & GS10 & 0.844 & 0.848 & 0.804 & 0.808 & 0.849 & 0.808 & 0.849 & 0.830 & 0.847 & 0.810 & 0.847 & 0.807 & 0.433 & \\
\hline 12 & M1SL & 0.712 & 0.714 & 0.867 & 0.823 & 0.755 & 0.722 & 0.711 & 0.713 & 0.751 & 0.827 & 0.713 & 0.719 & 1.709 & \\
\hline 13 & $\mathrm{M} 2 \mathrm{SL}$ & 0.759 & 0.757 & 0.672 & 0.671 & 0.760 & 1.290 & 0.762 & 0.690 & 0.781 & 0.704 & 0.759 & 0.668 & 0.957 & \\
\hline $14^{*}$ & UTL11 & 0.817 & 0.812 & 0.528 & 0.529 & 0.768 & 0.549 & 0.631 & 0.687 & 0.793 & 0.552 & 0.771 & 0.547 & 0.151 & $0^{2}$ \\
\hline $15^{*}$ & SP500 & 0.926 & 0.922 & 0.935 & 0.930 & 0.924 & 1.235 & 0.928 & 0.945 & 0.944 & 1.045 & 0.923 & 0.949 & 0.798 & 8 \\
\hline 16 & INDPRO & 0.925 & 0.923 & 1.062 & 1.034 & 0.935 & 0.911 & 0.992 & 0.963 & 0.970 & 1.011 & 0.980 & 0.925 & 1.035 & \\
\hline $17^{*}$ & HOUST & 0.844 & 0.849 & 0.794 & 0.791 & 0.851 & 0.805 & 0.663 & 1.003 & 0.863 & 0.851 & 0.851 & 0.806 & 0.090 & \\
\hline 18 & AHEMAN & 0.884 & 0.885 & 0.925 & 0.923 & 0.972 & 0.911 & 0.881 & 0.879 & 0.886 & 0.911 & 1.123 & 0.958 & 0.319 & U \\
\hline 19 & UNRATE & 0.966 & 0.966 & 0.967 & 0.969 & 0.976 & 0.942 & 1.117 & 1.135 & 1.001 & 1.063 & 0.959 & 0.974 & 0.235 & \\
\hline 20 & PAYEMS & 0.859 & 0.857 & 0.789 & 0.788 & 0.880 & 0.769 & 0.956 & 0.927 & 0.897 & 0.860 & 0.889 & 0.866 & 0.273 & 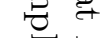 \\
\hline 21 & EXUSUK & 0.898 & 0.898 & 0.876 & 0.879 & 0.900 & 0.880 & 0.906 & 0.903 & 0.921 & 0.902 & 0.894 & 0.874 & 0.406 & (क) \\
\hline $22^{*}$ & PMI & 0.821 & 0.818 & 0.731 & 0.732 & 0.829 & 0.721 & 0.796 & 0.745 & 0.864 & 0.784 & 0.822 & 0.722 & 0.379 & \\
\hline $23^{*}$ & NAPMNOI & 0.866 & 0.863 & 0.823 & 0.821 & 0.864 & 0.801 & 0.879 & 0.820 & 0.909 & 0.892 & 0.863 & 0.799 & 0.568 & \\
\hline
\end{tabular}




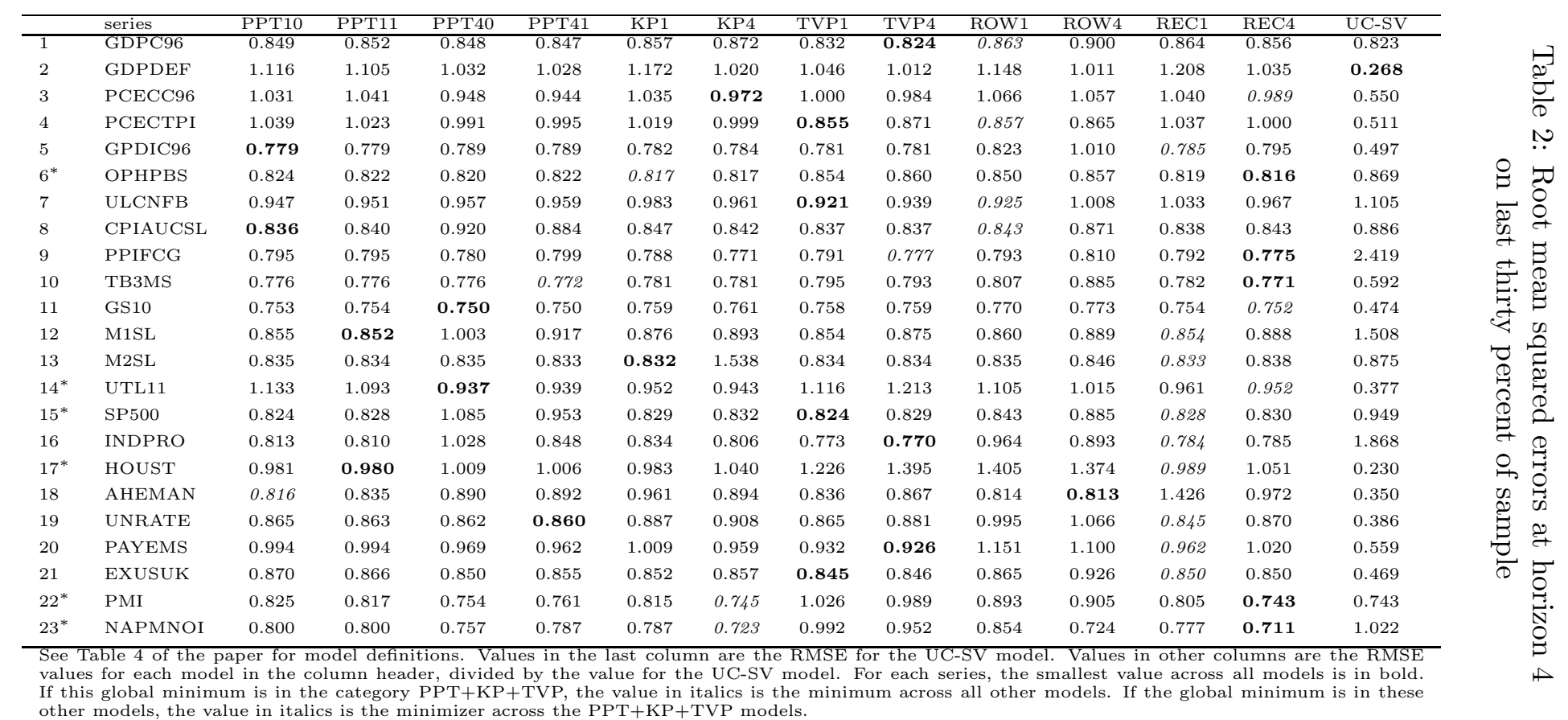




\begin{tabular}{|c|c|c|c|c|c|c|c|c|c|c|c|c|c|c|}
\hline & series & PPT10 & PPT11 & $\begin{array}{l}\text { PPT40 } \\
\end{array}$ & $\begin{array}{l}\text { PPT41 } \\
\end{array}$ & KP1 & KP4 & TVP1 & TVP4 & ROW1 & ROW4 & REC1 & REC4 & $\overline{\mathrm{UC}-\mathrm{SV}}$ \\
\hline 1 & GDPC96 & & 1.734 & & 1.663 & 1.008 & & 1.724 & 1.661 & & & & & \\
\hline 2 & GDPDEF & 3.068 & 3.019 & 2.858 & 2.821 & 2.970 & 2.957 & 2.060 & 2.085 & 3.306 & 3.280 & 2.505 & 2.630 & 0.423 \\
\hline 3 & PCECC96 & 1.415 & 1.403 & 1.488 & 1.474 & 1.441 & 1.607 & 1.528 & 1.692 & 1.783 & 1.932 & 1.425 & 1.487 & 0.333 \\
\hline 4 & PCECTPI & 2.264 & 2.247 & 2.408 & 2.391 & 2.370 & 2.476 & 1.893 & 1.916 & 2.704 & 2.586 & 2.328 & 2.490 & 0.388 \\
\hline 5 & GPDIC96 & 1.925 & 1.923 & 1.864 & 1.857 & 1.978 & 1.941 & 1.804 & 1.754 & 2.354 & 2.251 & 1.859 & 1.893 & 0.371 \\
\hline $6^{*}$ & OPHPBS & 1.509 & 1.502 & 1.551 & 1.552 & 1.521 & 1.576 & 1.575 & 1.533 & 1.626 & 1.583 & 1.525 & 1.588 & 0.230 \\
\hline 7 & ULCNFB & 1.403 & 1.408 & 1.370 & 1.355 & 1.337 & 1.393 & 1.454 & 1.479 & 1.563 & 1.492 & 1.275 & 1.375 & 0.218 \\
\hline 8 & CPIAUCSL & 2.200 & 2.201 & 2.333 & 2.303 & 2.287 & 2.422 & 1.927 & 2.068 & 2.519 & 2.608 & 2.282 & 2.499 & 0.288 \\
\hline 9 & PPIFCG & 1.558 & 1.561 & 1.597 & 1.599 & 1.582 & 1.666 & 1.738 & 1.675 & 1.508 & 1.545 & 1.615 & 1.690 & 0.157 \\
\hline 10 & TB3MS & 2.142 & 2.117 & 2.106 & 2.083 & 2.193 & 2.022 & 2.131 & 2.016 & 2.211 & 2.097 & 1.178 & 1.218 & 0.378 \\
\hline 11 & GS10 & 1.952 & 1.927 & 1.932 & 1.900 & 1.783 & 1.871 & 1.619 & 1.708 & 2.110 & 2.167 & 1.882 & 1.922 & 0.335 \\
\hline 12 & M1SL & 1.672 & 1.672 & 1.571 & 1.561 & 1.546 & 1.614 & 1.712 & 1.608 & 1.561 & 1.467 & 1.712 & 1.742 & 0.177 \\
\hline 13 & M2SL & 1.796 & 1.796 & 1.942 & 1.926 & 1.812 & 1.146 & 1.876 & 2.109 & 1.934 & 2.054 & 1.808 & 1.983 & 0.249 \\
\hline $14^{*}$ & UTL11 & 6.347 & 6.268 & 6.856 & 6.687 & 4.916 & 6.191 & 1.562 & 1.568 & 6.744 & 8.336 & 4.846 & 6.199 & 0.466 \\
\hline $15^{*}$ & SP500 & 1.626 & 1.616 & 1.564 & 1.567 & 1.645 & 1.571 & 1.528 & 1.520 & 1.608 & 1.556 & 1.634 & 1.588 & 0.268 \\
\hline 16 & INDPRO & 1.357 & 1.346 & 1.217 & 1.196 & 1.311 & 1.130 & 1.439 & 1.437 & 1.335 & 1.245 & 0.895 & 0.972 & 0.257 \\
\hline $17^{*}$ & HOUST & 7.475 & 7.386 & 7.583 & 7.548 & 7.558 & 7.811 & 1.587 & 1.523 & 9.639 & 9.497 & 7.603 & 7.818 & 0.485 \\
\hline 18 & AHEMAN & 2.552 & 2.519 & 2.266 & 2.240 & 2.361 & 2.255 & 1.772 & 1.856 & 2.550 & 2.513 & 1.616 & 1.941 & 0.374 \\
\hline 19 & UNRATE & 2.785 & 2.749 & 2.622 & 2.603 & 2.845 & 2.856 & 2.166 & 2.172 & 3.328 & 3.135 & 2.674 & 2.637 & 0.433 \\
\hline 20 & PAYEMS & 3.423 & 3.368 & 3.136 & 3.064 & 3.161 & 2.860 & 2.172 & 2.063 & 3.370 & 3.244 & 2.375 & 2.464 & 0.419 \\
\hline 21 & EXUSUK & 2.008 & 1.998 & 1.864 & 1.866 & 2.127 & 1.928 & 1.900 & 1.902 & 2.181 & 2.042 & 2.031 & 2.019 & 0.388 \\
\hline $22^{*}$ & PMI & 2.131 & 2.122 & 2.307 & 2.275 & 2.334 & 2.403 & 1.533 & 1.597 & 2.596 & 2.775 & 2.147 & 2.389 & 0.371 \\
\hline $23^{*}$ & NAPMNOI & 1.873 & 1.875 & 1.894 & 1.887 & 1.891 & 1.993 & 1.430 & 1.418 & 1.989 & 1.915 & 1.895 & 2.004 & 0.310 \\
\hline
\end{tabular}




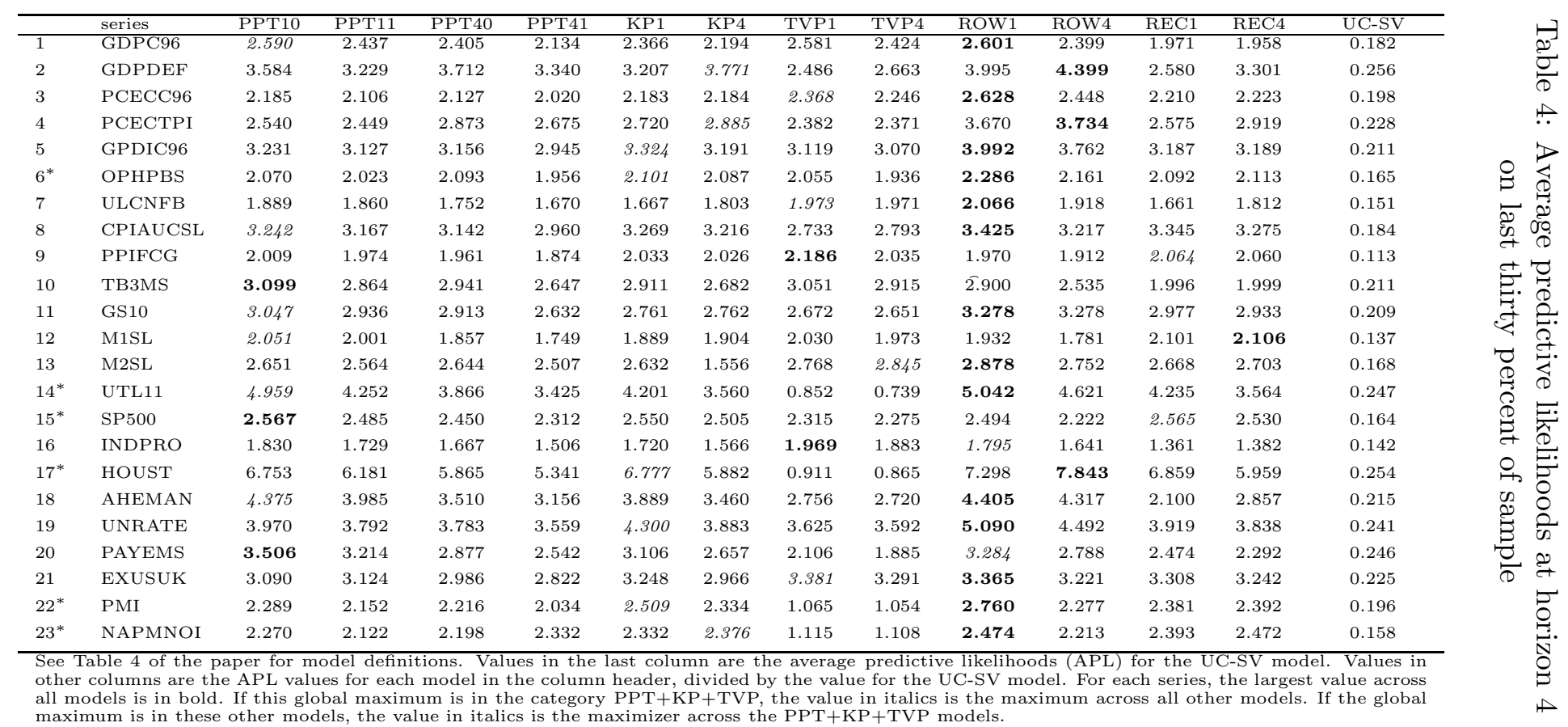




\section{References}

Bauwens, L., G. Koop, D. Korobilis, and J. Rombouts, 2011, The contribution of structural break models to forecasting macroeconomic series, Working paper available at http://personal.strath.ac.uk/gary.koop/.

Bauwens, L., and J. Rombouts, 2010, On marginal likelihood computation in change-point models, Computational Statistics \& Data Analysis in press. 